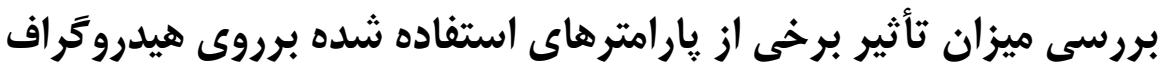 واحد لحظهاى زئومورفولوزيك (حوضه آبخيز آنيز كسيليان)
}

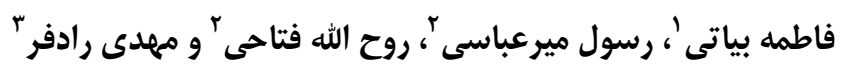

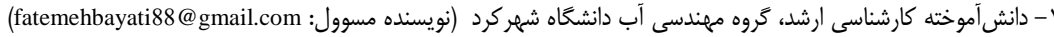

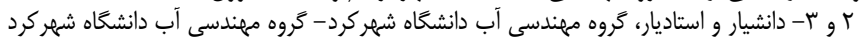

تاريخ دريافت:

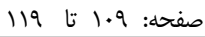

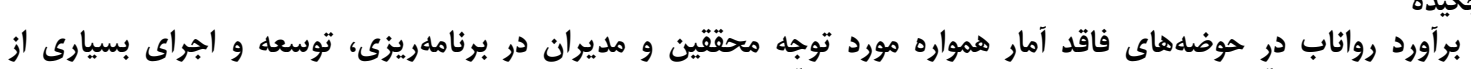

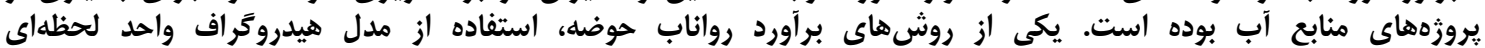

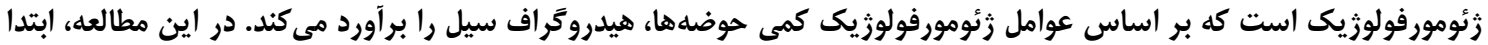

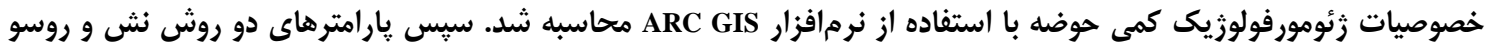

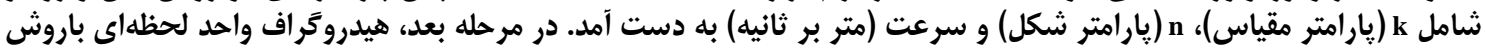

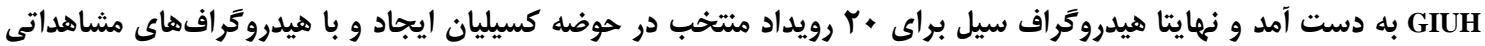

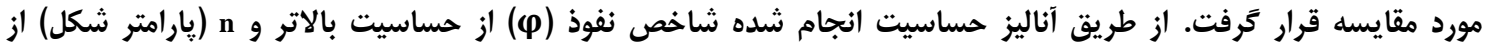

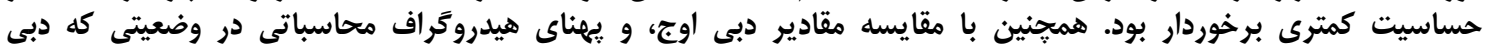

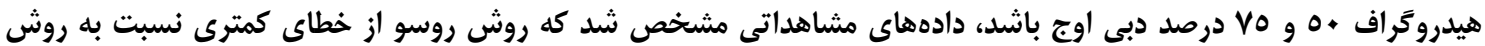

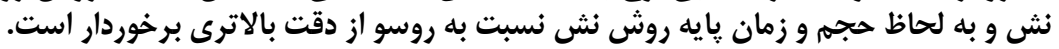

وازمهاى كليدى: آناليز حساسيت، يار امتر شكل، يارامتر مقياس، مدل GIUH، هيدروتراف

كيلومتر مربع، هيدروكراف واحد استاندارد را ارائه كرد.

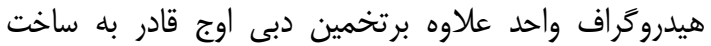

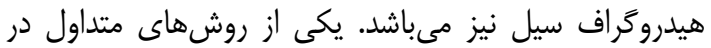

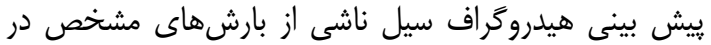

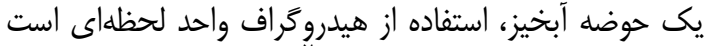

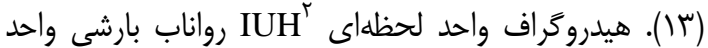

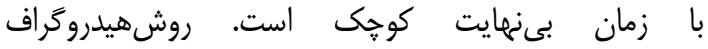

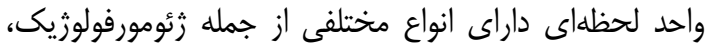

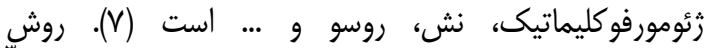

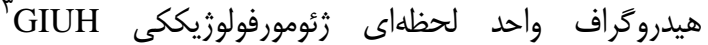

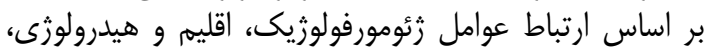

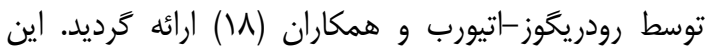

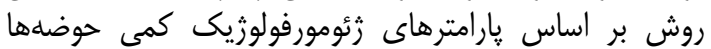

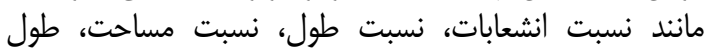

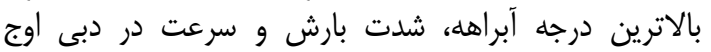

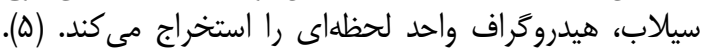

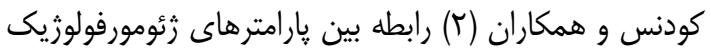

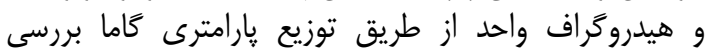

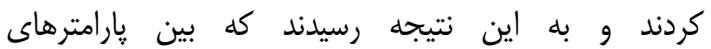
زئومورفولوزيك حوضه آبريز و واساسخ هيدرولوزيك آنى بارئ رابطه

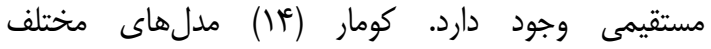

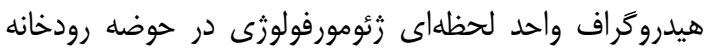

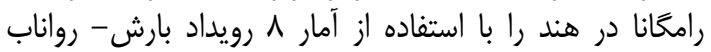

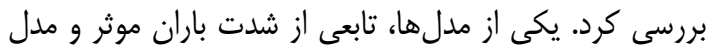

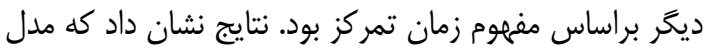

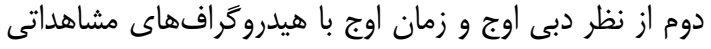

مقدمه

سيل يكى از عمدهترين مسائل بحرانى محيط زيست و و مقات حتى جوامع بشرى است. تحقيقات متعددى در در راستاى شناخت

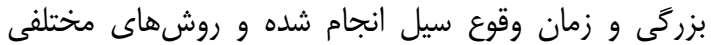

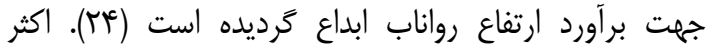

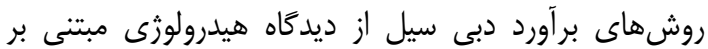

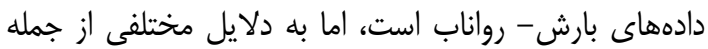

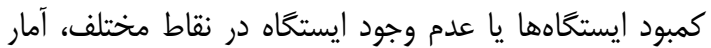

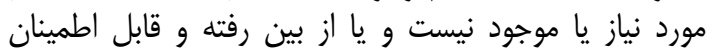

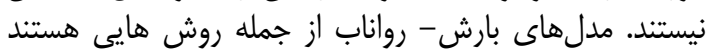

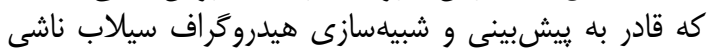

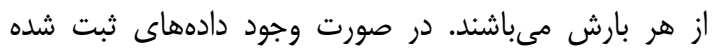

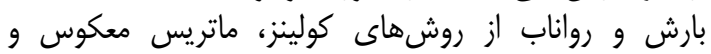

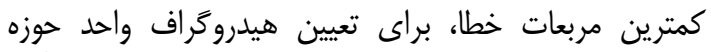

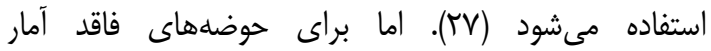

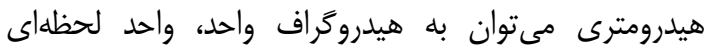

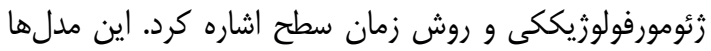

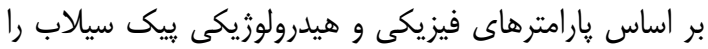

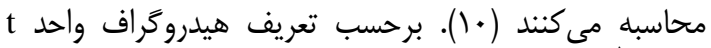

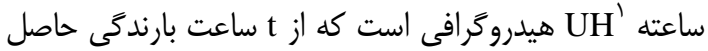

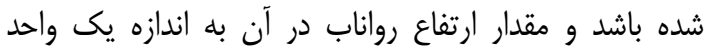

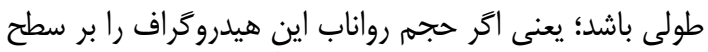

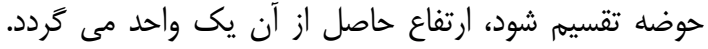

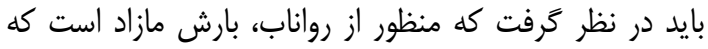

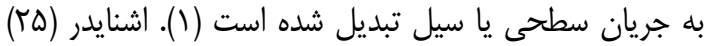

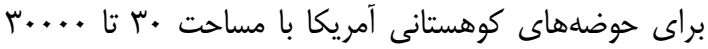


حوضه ماتريس همبستى برقرار كردند و از انواع معادلات

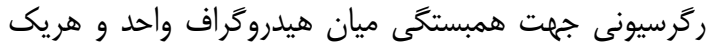

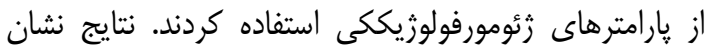

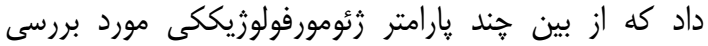

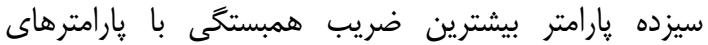

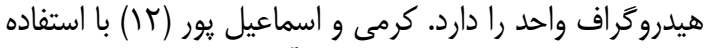

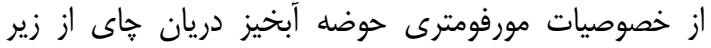

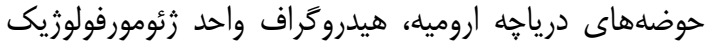

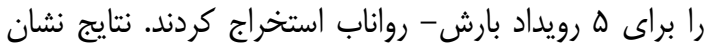

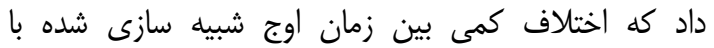

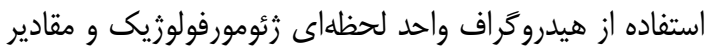

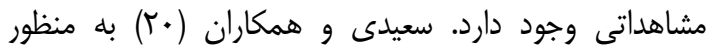
بررسى هيدروكراف و واحد ل لحظهاى

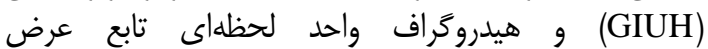

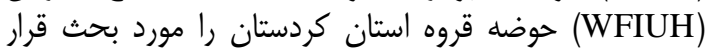

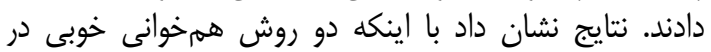

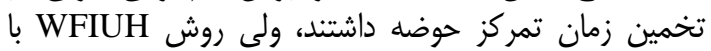

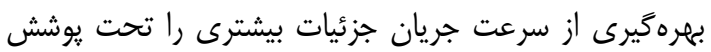

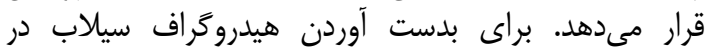

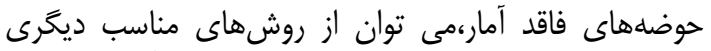

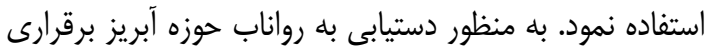

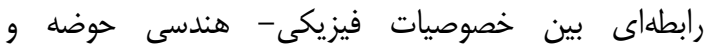

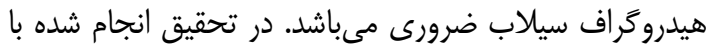

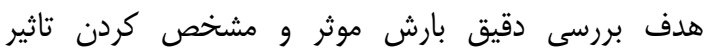

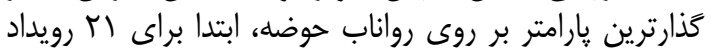

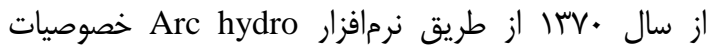

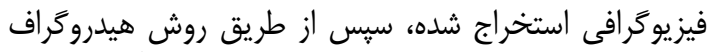

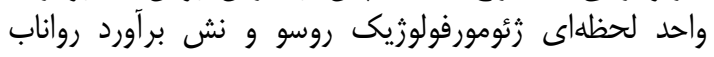

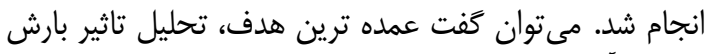

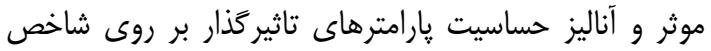

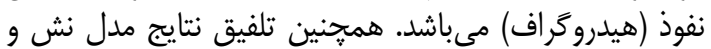

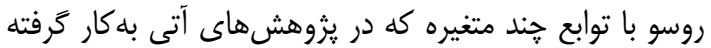
شدهاند، مىتوانند از جمله اهداف اين يزوهش بردي باشد.

\section{مواد و روشها - موشيا}

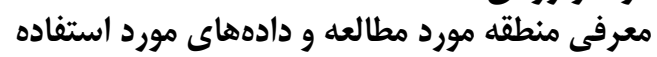

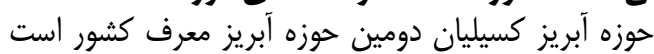

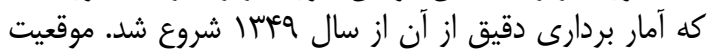

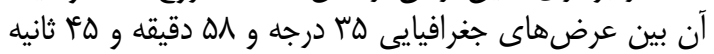

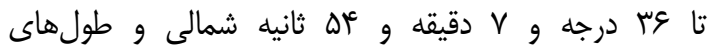

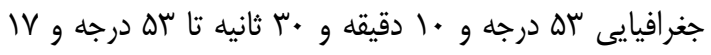

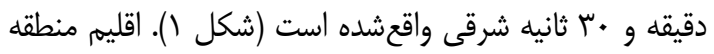

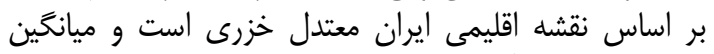

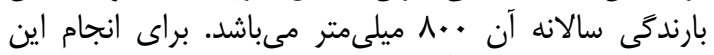

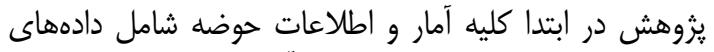

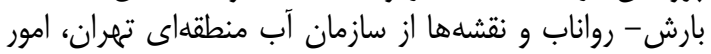

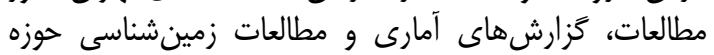

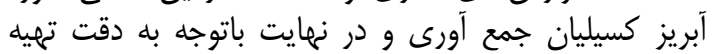
آنها، جزئيات مورد نظر و همجنين همزمانى با بارش به به وقوع
همبستكى بهترى دارد. ايسكندرا و ساجى كومار (•) به به به

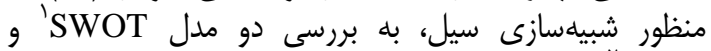

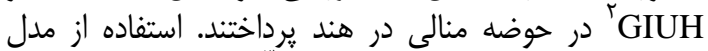

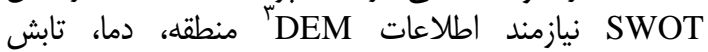

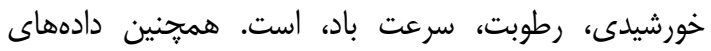

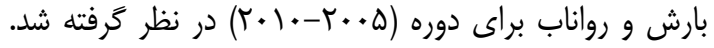

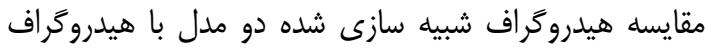

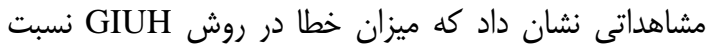

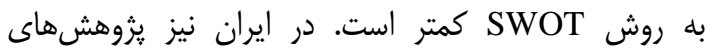

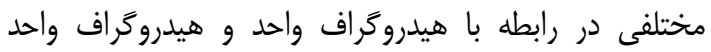

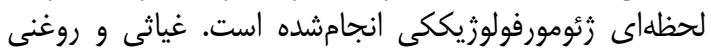

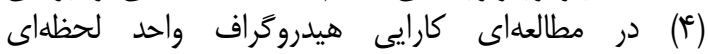

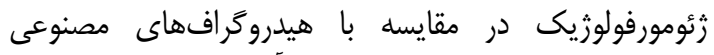

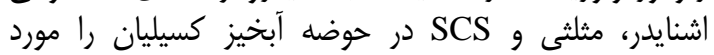

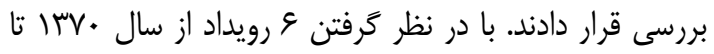

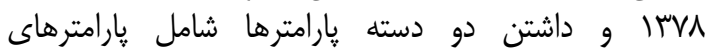

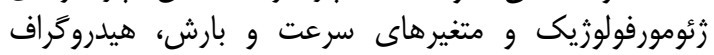

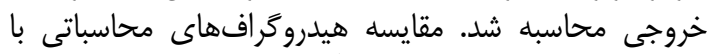

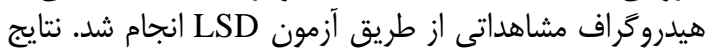

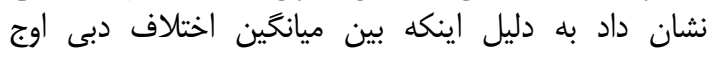

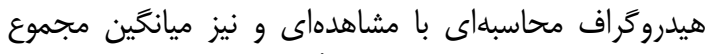

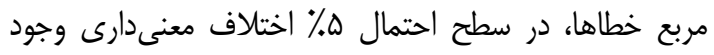

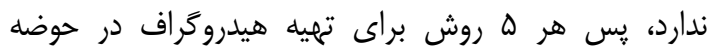

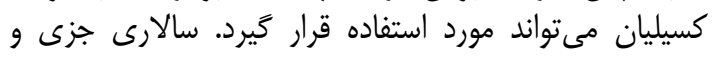

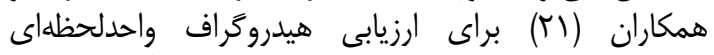

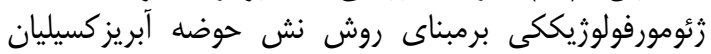

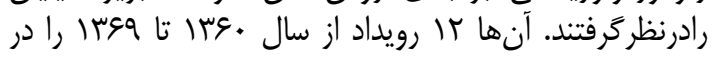

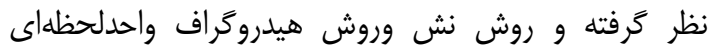

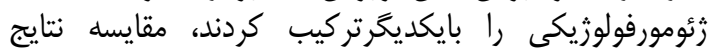

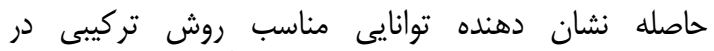

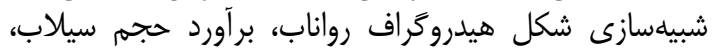

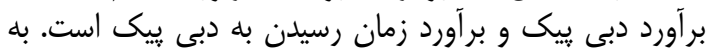

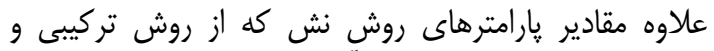

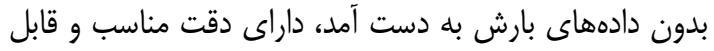

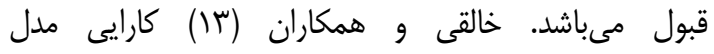

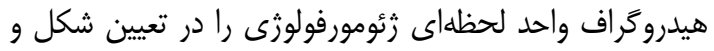

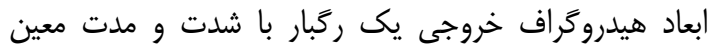

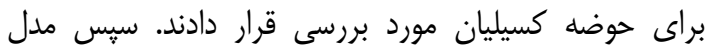

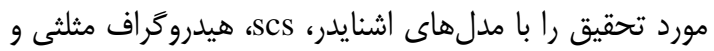

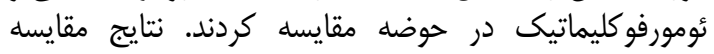

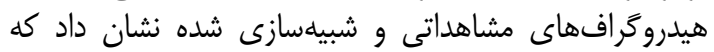

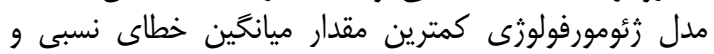

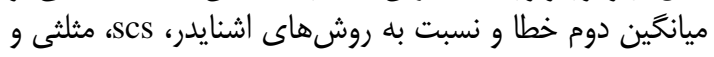

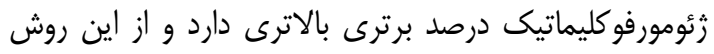

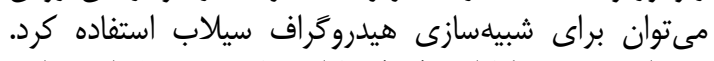

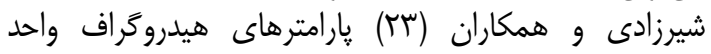

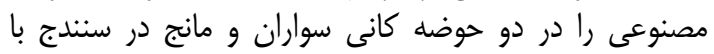

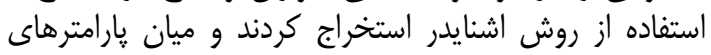

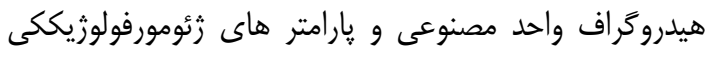


براى هر واقعه سيل، داشتن هيدروكراف و تاج كاملاً مشخص

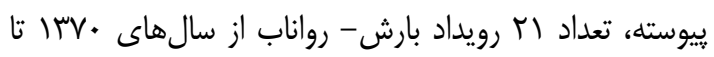

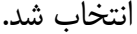

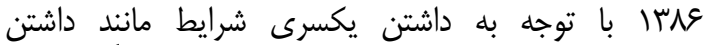
هايتوكراف متناظر سيل، داشتن ابتدا و انتهاى كاملاً مشخص انش
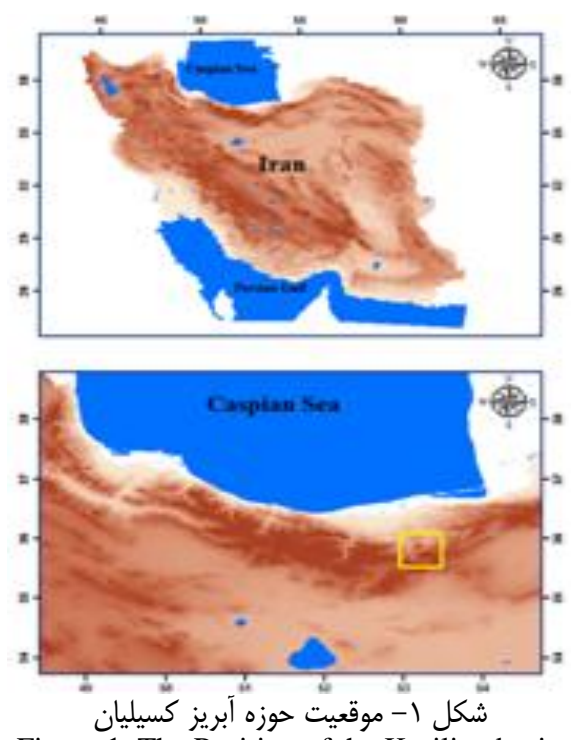

Figure 1. The Position of the Kasilian basin

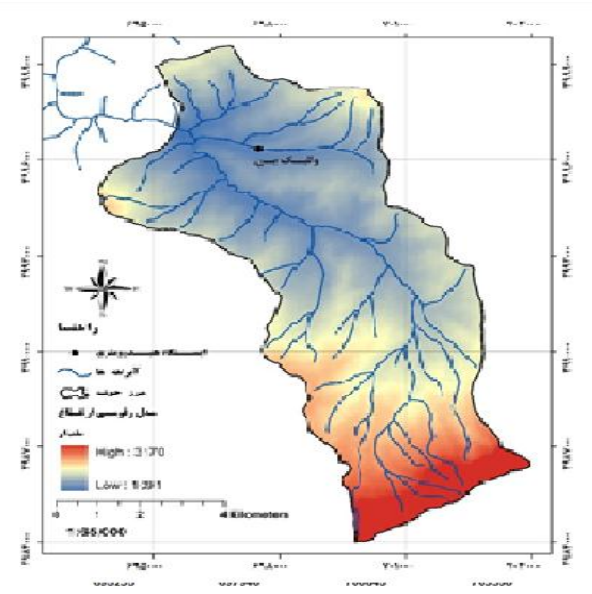

شكل r- نقشه DEM حوزه آبريز كسيليان

Figure 2. DEM Map of the Kasilian basin

در محيط Watershed Processing و Preprocessing خصوصيات فيزيوكرافى حوضه آبخيز ماندا

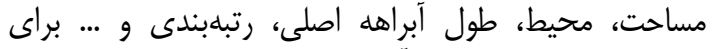

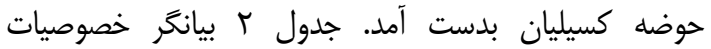

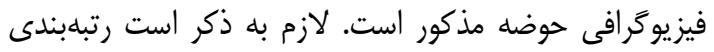

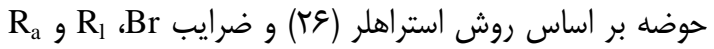
طبق قوانين هورتن (ᄉ) محاسبه شده است.
تعيين خصوصيات فيزيوترافى حوزه آبخيز با استفاده از

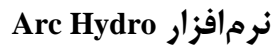
نرمافزار Arc Hydro ساختار دادهاى در GIS است كه

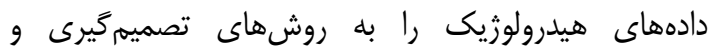

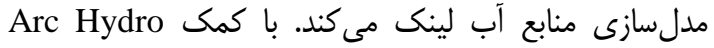

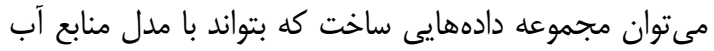

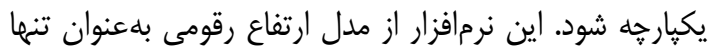

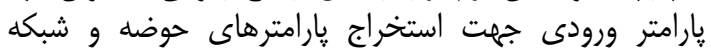
آبراهلهاى استفاده مى كند. با استفاده از دو دستور 


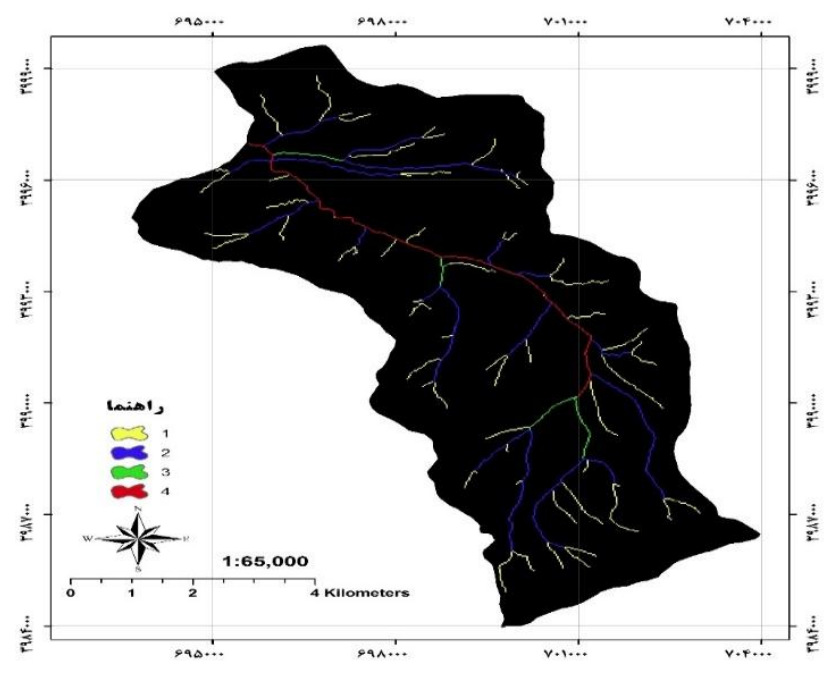

شكل r- مشخص شدن رتبلبندى آبراهdهاى حوضه كسيليان

Figure 3. Determination of the ranking of the channels of the Kasilian Basin

Table 1. Caracteristics and Ratings of the Kasiliancathments

جدول 1- مشخصات و رتبهبندى آبراهdهاى حوزه آبريز كسيليان

\begin{tabular}{|c|c|c|c|c|c|c|c|c|c|c|}
\hline$R_{a}$ & $R_{l}$ & $B_{r}$ & 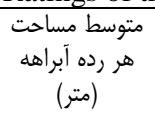 & هورل آبراهـه & $\frac{N_{i}}{N_{i+1}}$ & انشعابات & آبراهد & طول زهك (متر) & محيط حوضه & (متر مربع) \\
\hline \multirow[t]{4}{*}{$T / T V$} & $r / \mu$ & T/GT & एАКчя... & $19 .$. & $r / \Lambda)$ & FT & 1 & 19T.. & 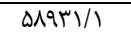 & 994NFDQ9 \\
\hline & & & arqf... & iva. & r/9 & 11 & r & & & \\
\hline & & & GTMED... & $r \cdot V \Delta$. & r & r & r & & & \\
\hline & & & g $\vee \wedge . . .$. & 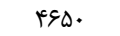 & & 1 & r & & & \\
\hline
\end{tabular}

$U(t)=\frac{1}{k \Gamma_{n}}\left(\frac{t}{k}\right)^{n-1} e^{-\frac{(t)}{k}}$

كه در آن، $(t)$ آن از صفر تا بىنهايت برابر با با يك است است. مؤلفه

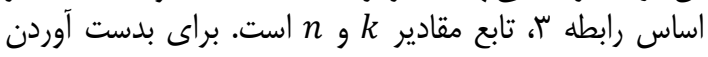
:ارامترهاى مدل نش از روابط زير استفاده شدهاست: $M Q_{1}-M I_{1}=n \times k$

$M Q_{2}-M I_{2}=n(n+1) k^{2}+2 n k M I_{1}$

كه در آن، اول و دوم هيدروگراف رواناب و هيتو مبدأ مىباشند.

هيدروكراف واحد لحظداف زئومورفولوزيكى بر مبناى روش نش

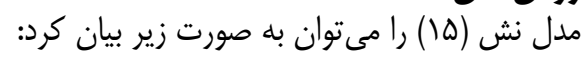
$U(0 . t)=\frac{1}{k \Gamma_{n}}\left(\frac{t}{k}\right)^{n-1} e^{-\frac{(t)}{k}}$

كه در آن، (O.t)

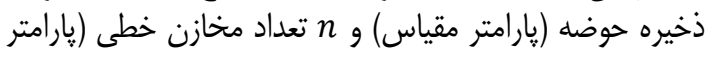

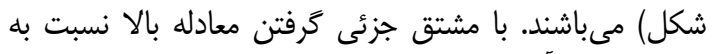

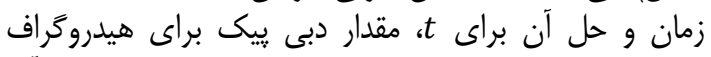

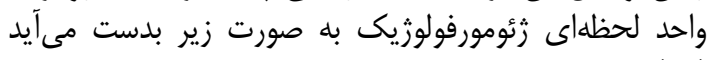

$q_{p}=\frac{1}{\Gamma_{n}} e^{-(n-1)}(n-1)^{n-1}$

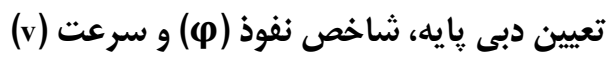

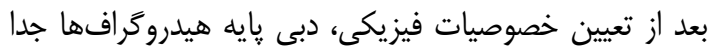

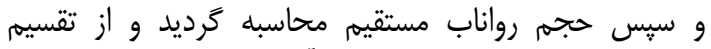

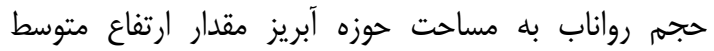

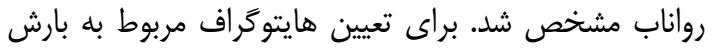

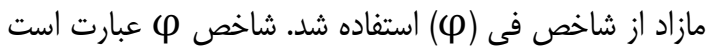

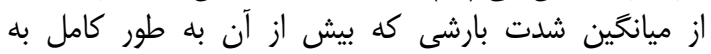

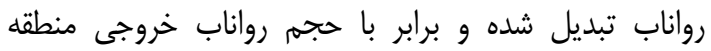
$\varphi=\frac{P-R}{t}$

ه در آن، P مجموع ارتفاع بارش (ميلىمتر)، R ارتفاع رواناب

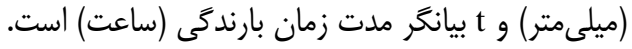

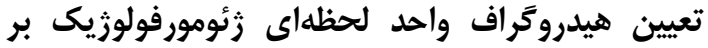

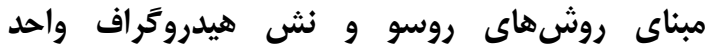
لحظهاى نش مبناي رون نش (ها) درمدل مفهومى خود، يك حوزه آبريز را به مثابه

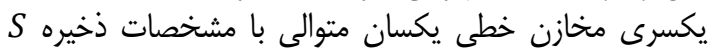

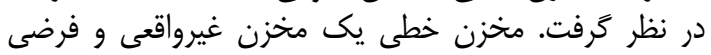
$S=K Q$ است كه با دبى خروجى رابطه مستقيه خطى دئ دارد:

در اين رابطه، $K$ ثابت مخزن يا ضريب ذخيره ناميده مي شود

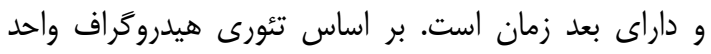

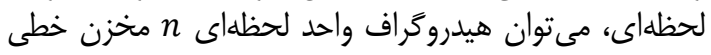

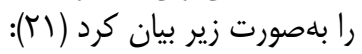




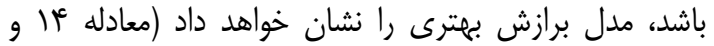

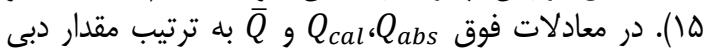

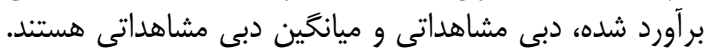

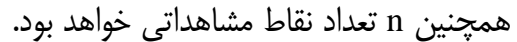

$N_{S}=1-\frac{\sum_{i=1}^{n}\left(Q_{a b s}-Q_{c a l}\right)^{2}}{\sum_{i=1}^{n}\left(Q_{a b s}-\bar{Q}\right)^{2}}$

$R M S E=\sqrt{\frac{\sum_{i=1}^{n}\left[\left(Q_{c a l}-Q_{a b s}\right)\right]^{2}}{n}}$

\section{بحث و نتايج}

به جهت مقايسه دو روش نش و روسو ابتدا مقدار شاخص

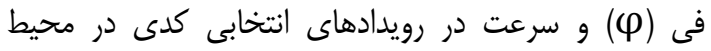

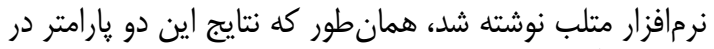

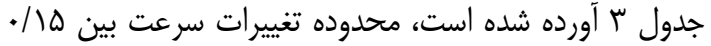

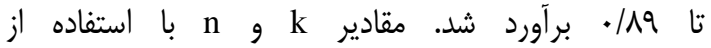

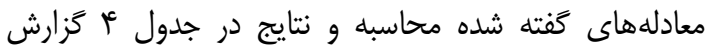

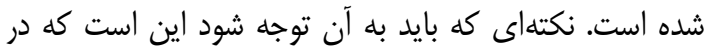

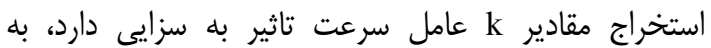

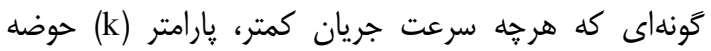
بيشتر خواهد شد. بعد از محاسبه اين بارارامترها، هيدروكراف براف

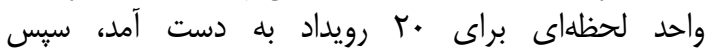

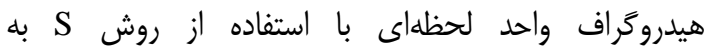

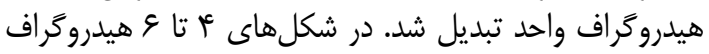

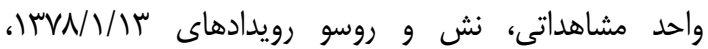

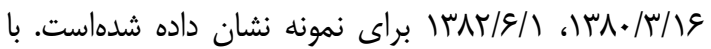

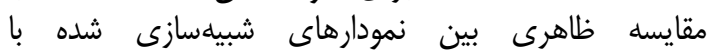
هيدروكرافهاى مشاهداتى مىتوان مشان مشاهداه كرد كه مداري

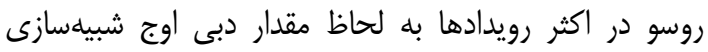

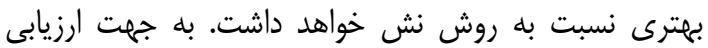

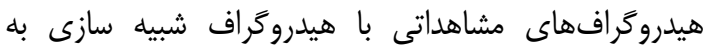

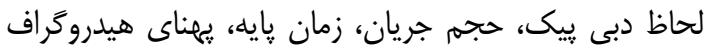

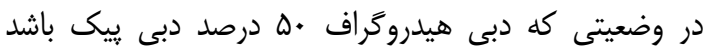

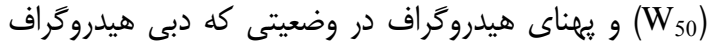

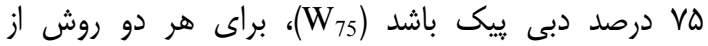

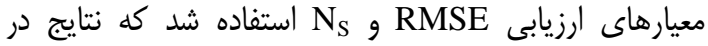
جدول ه ارائه شده است. $q_{p} \cdot t_{p}=\frac{(n-1)}{\Gamma_{n}} e^{-(n-1)}(n-1)^{n-1}$

كه در آن، $q_{p}$ دبى اوج (بر ساعت) و t زمان زمان اوج (ساعت)

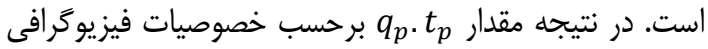

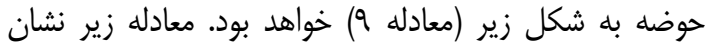

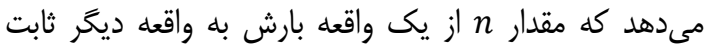

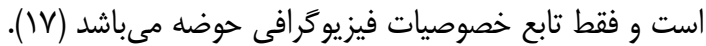

$\frac{(n-1)}{\Gamma_{n}} e^{-(n-1)}(n-1)^{n-1}=$

$0.5746 R_{B}^{0.55} R_{A}{ }^{-0.55} R_{L}^{0.05}$

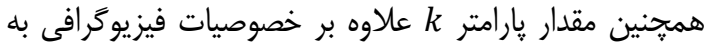

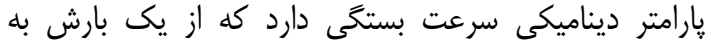

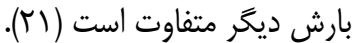

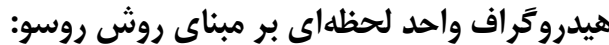

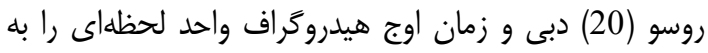

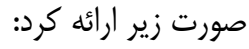

$q_{p}=0.364 R_{L} L_{\Omega}^{-1} \cdot V$

$t_{p}=1.584\left(\frac{R_{B}}{R_{A}}\right) \cdot 55 R_{L}^{-0.38} L_{\Omega} V^{-1}$

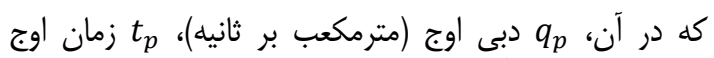

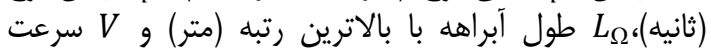
جريان (متر بر ثانيه) مى باشد. مقدار از معادلات زير به دست مى آيند:

$k=0.7\left(\frac{R_{A}}{R_{B} \cdot R_{L}}\right)^{0.48} \cdot L_{\Omega} \cdot V^{-1}$

$n=3.29\left(\frac{R_{B}}{R_{A}}\right)^{0.78} \cdot R_{\mathrm{L}}^{0.07}$

كه در آن،

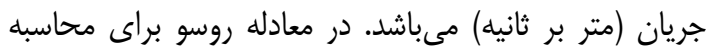

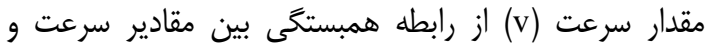
دبى براى هر رويداد استفاده شد.

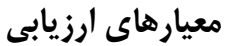

به جهت مقايسه بين نتايج بدست آمده در دو روش نش و وريار

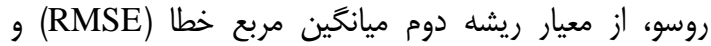

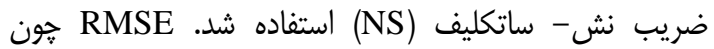

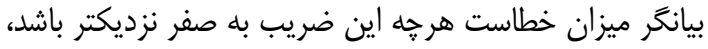

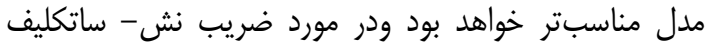

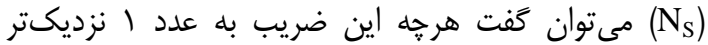


Table 2. Prameters of speed and intrusion index for each event

جدول r- يارامترهاى سرعت و شاخص نفوذ براى هر رويداد به كار گرفته شده

\begin{tabular}{|c|c|c|c|c|c|}
\hline سرعت (متر بر ثانيه) & شاخص نفوذ & رويداد & سرعت(متر بر ثانيه) & شاخص نفوذ & رويداد \\
\hline 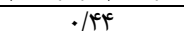 & $F / 49$ & |TMT/g/I &.$/ 199$ & $V / f^{2}$ & $|r v / r / \mu|$ \\
\hline 吾/ & $.1 \cdots 10$ & IrAr/v/Ir & .199 & .1 .1 & Irv/v/s \\
\hline س &.$/ 4 \Delta$ & س/r/r/m &.$/ 49$ & $1 / r q$ & ITVT/T/IQ \\
\hline ת.r/\% & .1 .0 & 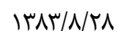 &.$/ 0$ &.$/ 9 V$ & ITVT/F/Tr \\
\hline.$/ 9 V$ & .1 .10 & |r &.$/ T \Delta$ & $1 / \mu 1$ & $1 \Psi r / q / 9$ \\
\hline.$/ \mu \Delta$ & $.1 . .90$ & IrNF/N/IQ & $\cdot / N$ & $8 / 1$ & $\mid r V+/ T / T V$ \\
\hline - / $A \Delta$ & תז/. & IrNF/N/IV & .109 &.$/ 995$ & ITVG/T/L \\
\hline | I & .149 & ITNه/G/KG &.$/ 19$ & $\wedge$ & $\mid r V G / F / T$ \\
\hline ITA &.$|\ldots|$ & /rNه//1//9 & .190 & . & Tr/M \\
\hline \multirow[t]{2}{*}{$. / g+\Lambda$} & $.1 \cdot 19$ & $\mid r / \Delta / T / 1$. & - /Ar & .119 & $1 \% v q / N / q$ \\
\hline & & &.$/ \Delta$ &.$/ 1$ & 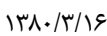 \\
\hline
\end{tabular}

جدول س- مقادير پارامترهاى k و براى هر دو روش نش و روسو

Table 3. The values of the parameters $\mathrm{n}$ and $\mathrm{k}$ for both Nash and Rosseau methods

\begin{tabular}{|c|c|c|c|c|c|c|c|c|c|}
\hline \multicolumn{2}{|c|}{ روش نش } & \multicolumn{2}{|c|}{ روش روسو } & \multirow[t]{2}{*}{ رويداد } & \multicolumn{2}{|c|}{ روش نش } & \multicolumn{2}{|c|}{ روش روسو } & \multirow[t]{2}{*}{ رويداد } \\
\hline $\mathrm{n}$ & $\mathrm{k}$ & $\mathrm{n}$ & $\mathrm{k}$ & & $\mathrm{n}$ & $\mathrm{k}$ & $\mathrm{n}$ & $\mathrm{k}$ & \\
\hline$F / \mu \wedge$ & $\mathrm{m} / 11$ & $p / r q$ & $\mathrm{~m} / \mathrm{s}$ & $\mid r \Delta r / \varepsilon / 1$ & $\Lambda / V \pi$ & $r / Y \Lambda$ & $p / \% q$ & $r / \cdot 1$ & |r/m/r \\
\hline F/r & $m / M$ & $p / r q$ & $1 / 9 V$ & IrNT/v/I & $p / 91$ & V/AT & $p / r_{q}$ & $r / \cdot 1$ & $|r v| / v / 19$ \\
\hline $11 / 11$ & سT/T & $p / r q$ & T/GT & س/ & Tr/ & $r / 9 \Lambda$ & $r / \% q$ & $r / \uparrow \wedge$ & $\mid r V T / r / 1 \Delta$ \\
\hline $0 / .9$ & D/qT & $p / \mu q$ & $r / \Delta q$ & 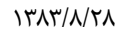 & $\mathrm{V} / \cdot \mathrm{\Lambda}$ & $r / r q$ & $p /$ qq & $r / f$ & ITVT/F/Tr \\
\hline$\mu / \mu$ & $T / T V$ & $p / r q$ & $r / \cdot V$ & $\mid r \wedge r / q / \kappa$ & $r / I T$ & ه/AT & $p /$ rq & $r / \Lambda$ & $1 \pi v / q / 9$ \\
\hline سוو & T/AT & $p / r q$ & $r / q$ & IrNF/N/ID & $1 \cdot / 4$ & $1 / 95$ & q q q & $r / T)$ & $\mid r V c / r / T V$ \\
\hline س/D/ & $r / r q$ & $p / r q$ & س & IrNF/N/IV & $q / p q$ & $r / \Delta \Lambda$ & $p / \mu_{q}$ & $r / \& V$ & IrVG/T/Ig \\
\hline r/gF & $V / \Delta$ & $p / r q$ & $N / F^{+}$ & $\mid r \wedge \Delta / \& / T \&$ & 1/99 & $T / D \mid$ & $p / r q$ & $1 / \Delta \xi$ & IrVG/r/IT \\
\hline$V / f$ & $r / \cdot 1$ & $p / r q$ & r/qv & $1 \%$ Ma/11/19 & $\Delta / G F$ & $\Gamma / \Delta \Delta$ & q/א & $8 / 91$ & سו/Ir/M \\
\hline s/ar & $r / 9$ & $p / r q$ & $r / \cdot r$ & $\mid r \wedge \Delta / T / 1$. & $r / V T$ & $r / 9 \mid$ & $p / r_{q}$ & $1 / 8 \Delta$ & $17 v q / 1 / q$ \\
\hline & & & & & $\Gamma / \Delta$ & سו/ه & $p / q q$ & $r / V \Lambda$ & \\
\hline
\end{tabular}

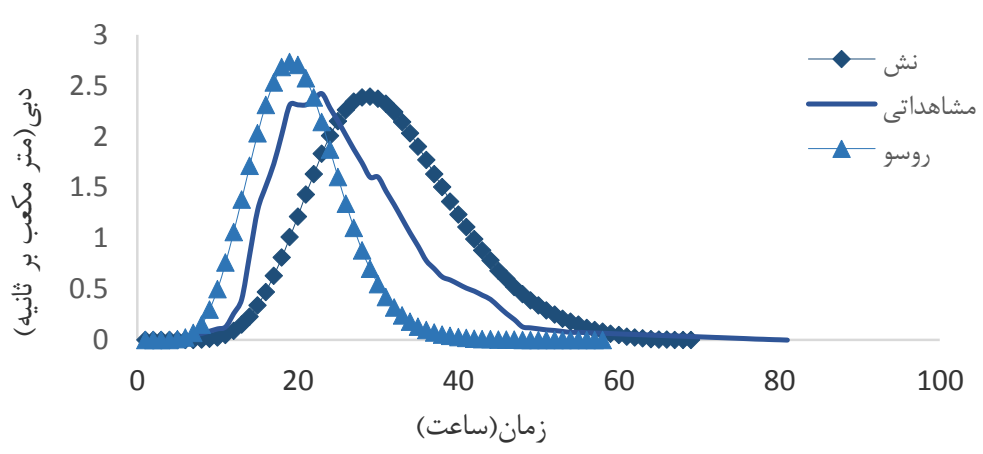

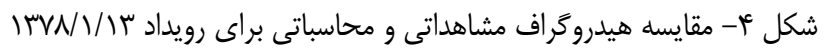

Figure 4. Comparison of observational and computational hydrographs for the event 1999/04/02

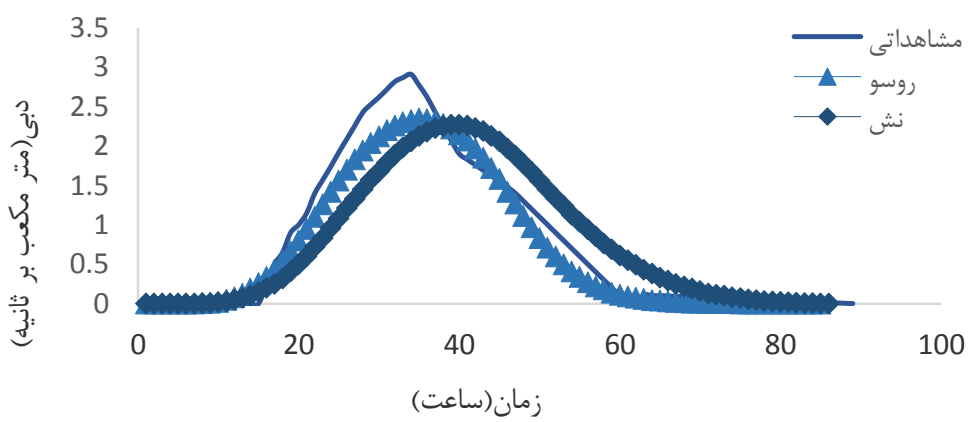

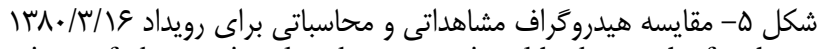

Figure 5. Comparison of observational and computational hydrographs for the event 2001/05/24 


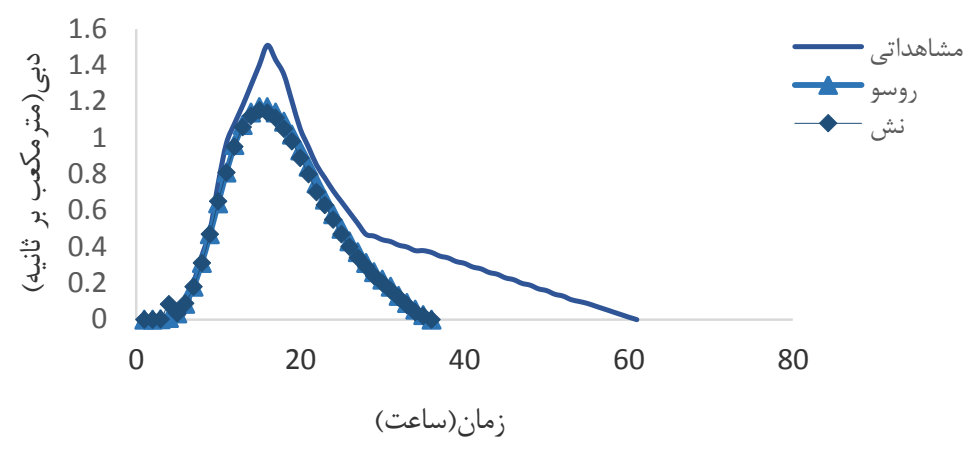

شكل \&- مقايسه هيدروكراف مشاهداتى و محاسباتى براى رويداد / / /

Figure 6. Comparison of observational and computational hydrographs for the event 2003/08/28

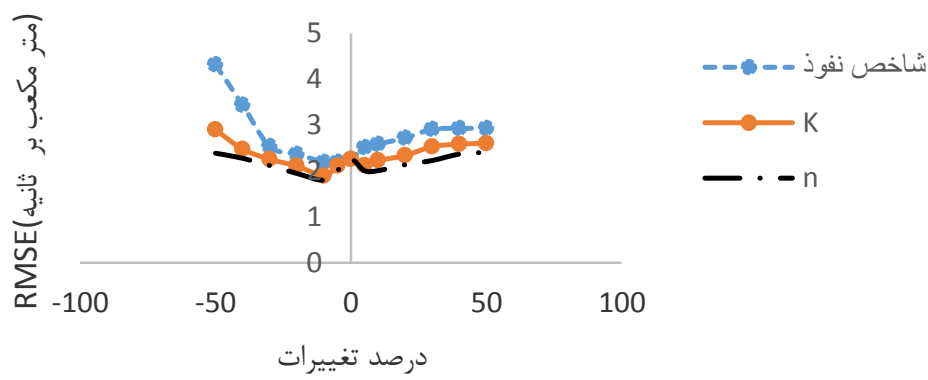

شكل V- نمودار آناليز حساسيت براى هيدروگراف واحد لحظهاى ثئومورفولوزى

Figure 7. Sensitivity analysis diagram for Geomorphologic Instantaneous unit htdrograph

Table 4. The result of fitting by sample kolmogrove- smirnov

$$
\text { جدول ع- نتايج آزمون نكويى برازش به روش كولموكراف- اسميرنوف }
$$

\begin{tabular}{|c|c|c|c|c|}
\hline & & Observe & GIUH- Rousseau & GIUH-Nash \\
\hline $\mathrm{N}$ & & $r+$ & $r$. & $r$ r. \\
\hline \multirow{3}{*}{ Normal Parameters ${ }^{\mathrm{a}}$} & Mean & 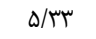 & $r / \mu$ & $r / q$ \\
\hline & Std. Deviation & $\Delta / V T$ & $1 / 98$ & $r / \mathscr{T V}$ \\
\hline & Absolute & $\cdot /$ TIA & $\cdot / r \Delta \Lambda$ & $\cdot / t \cdot t^{c}$ \\
\hline \multirow[t]{2}{*}{ Most Extreme Differences } & Positive & 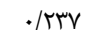 & $\cdot / r \Delta \Lambda$ & $\cdot / t+r^{c}$ \\
\hline & Negative & $-\cdot /$ TFA & $-T / T V$ &.$- / 1 V 9$ \\
\hline Kolmogorov-Smirnov Z & & $1 / 1 \cdot 1$ & I/IOT &.$/ 914$ \\
\hline Asymp. Sig. (2-tailed) & &.$/|V|$ & $.|| f \mid$ & $\cdot / \mathrm{rVV}$ \\
\hline
\end{tabular}

جدول ه- نتايج آزمون t مستقل براى دبى اوج با دو روش هيدروگراف واحد لحظه اى زئومورفولوزيك روسو و نش

Table 5. Independent of t-test for peak discharge with two methods of instantaneous Geomorphologic Instantaneous Unit Hydrograph of Rousseau and nash

\begin{tabular}{|c|c|c|c|c|}
\hline \multirow[b]{2}{*}{ روشهاى مورد استفاده } & \multicolumn{4}{|c|}{ t-test for Equality of Means } \\
\hline & $\mathrm{F}$ & Std Error Difference & Sig(2-tailed) & Mean Diffrence \\
\hline هيدروگراف واحد لحظهاى زئومورفولوزيكى روسو & $\cdot / 4 \wedge 1$ & $1 / 90$ & $\cdot / \Delta M_{q}$ & $1 / \cdot r$ \\
\hline 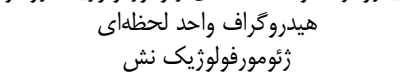 & $1 / \Delta$ & I/DT & - /raf & Jet \\
\hline
\end{tabular}

جدول צ- نتايج آزمون t مستقل براى زمان تا اوج با دو روش هيدروگراف واحد لحظه اي زئومورفولوزيك روسو و نش

Table 6. Independent of t-test for time to peak with two methods of instantaneous Geomorphologic Instantaneous Unit Hydrograph of Rousseau and nash

\begin{tabular}{|c|c|c|c|c|}
\hline \multirow[b]{2}{*}{ روشهاى مورد استفاده } & \multicolumn{4}{|c|}{ t-test for Equality of Means } \\
\hline & $\mathrm{F}$ & Std Error Difference & Sig(2-tailed) & Mean Diffrence \\
\hline هيدرومَراف واحد لحظهاى & $\cdot / \cdot \cdot V$ & $r / g t$ & ./194 & $-\cdot / \Delta$ \\
\hline 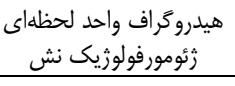 & 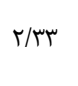 & 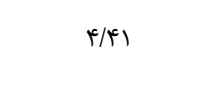 & $\cdot / 4 \cdot 1$ & $-r / V \Delta$ \\
\hline
\end{tabular}


Table 7. RMSE and N $\mathrm{N}_{S}$ evaluation criteria for both Nash and Rousseau

\begin{tabular}{|c|c|c|c|c|c|c|c|c|c|c|}
\hline \multicolumn{2}{|c|}{ (hr) W75 } & \multicolumn{2}{|c|}{$(\mathrm{hr}) \mathrm{W} 50$} & \multicolumn{2}{|c|}{ زمان پايه (hr) } & \multicolumn{2}{|c|}{ حجم $\left(\mathrm{m}^{3}\right)$} & \multicolumn{2}{|c|}{ دبى ييك (m³) } & \multirow[t]{2}{*}{ معيار ارزيابى } \\
\hline نش & روسو & نش & روسو & نش & روسو & نش & روسو & نش & روسو & \\
\hline$F / \Delta q$ & $T / T$ & $N / r$ & $r / q 1$ & $\mid r / r \Delta$ & $\mid q / 8$ & $r / 9 V$ & $r / M$ & $r / .9$ & $T / 4 G$ & RMSE \\
\hline.$- / 9 V$ & $\cdot / D F$ & $-1 / \cdots \Delta$ &.$/ D F$ &.$/ T V$ & سו/.- & $\cdot|\lambda|$ & $\cdot / \mathrm{VA}$ &.$/ 9 \mathrm{~V}$ & ./AT & $\mathrm{N}_{\mathrm{S}}$ \\
\hline
\end{tabular}

رسيدن به دبى اوج كافى نيست، بلكه نياز است از روشهاى تركيبى استفاده كرد.

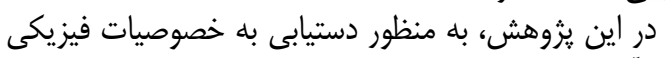

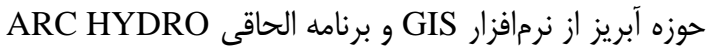

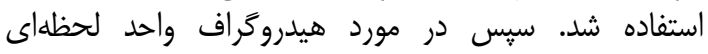
زئومورفولوزيك از دو روش روش نش و رور روسو استفاده شد.

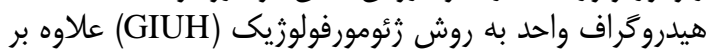

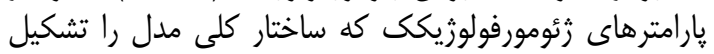

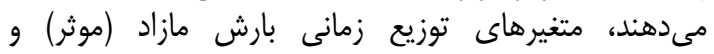

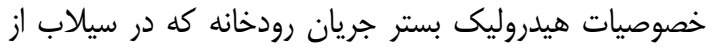

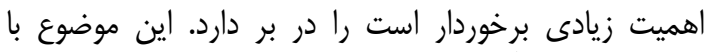
نتايج به دست آمده از تحقيقـات خالقى و و همكاراران (سارا)،

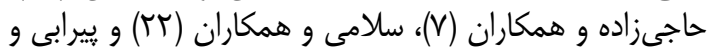

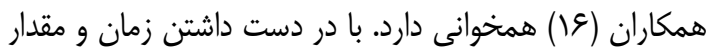

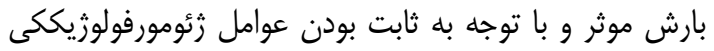

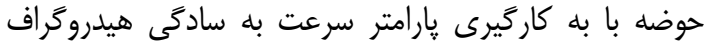

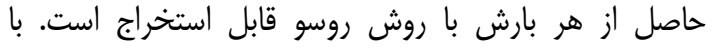

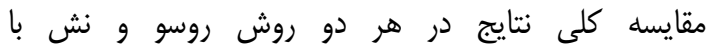

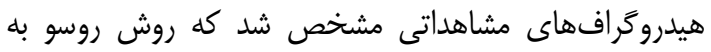

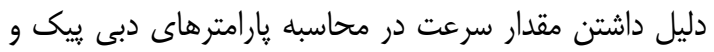

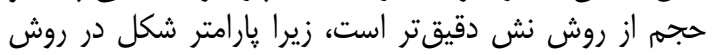

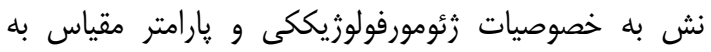

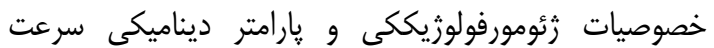

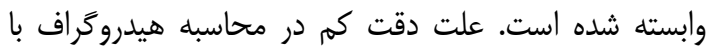

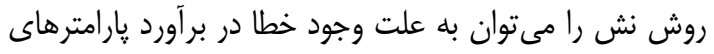

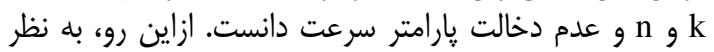

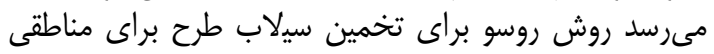

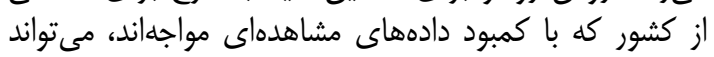

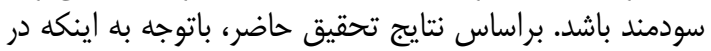

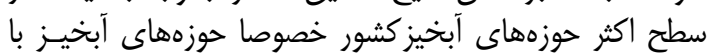

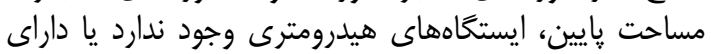

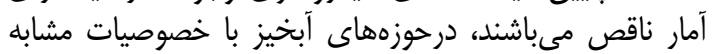

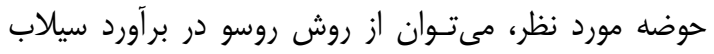

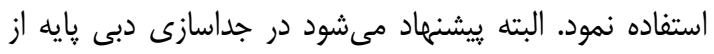

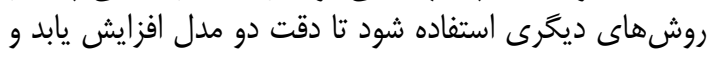

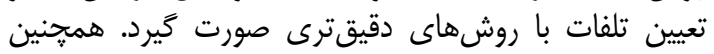

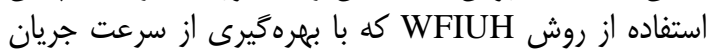

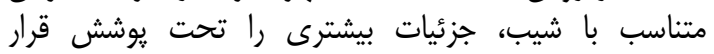
مى مهد، توصيه مىشود.

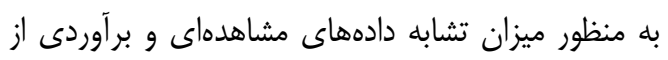

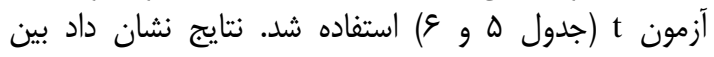

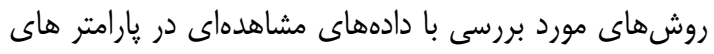

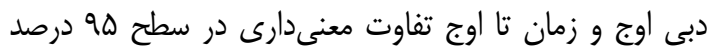

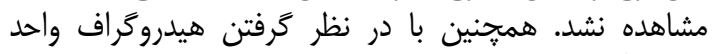

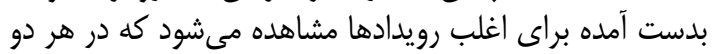

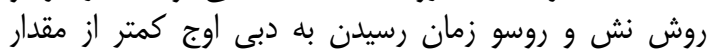

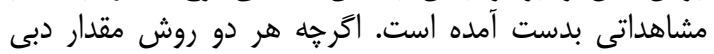

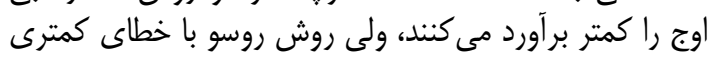

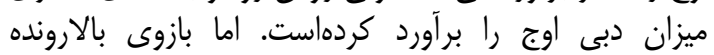

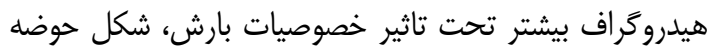

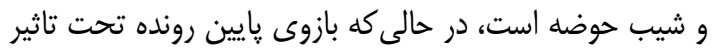

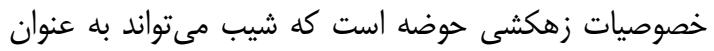

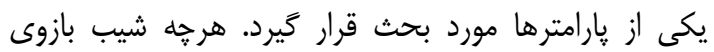

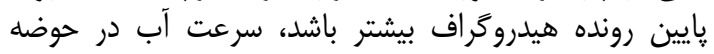

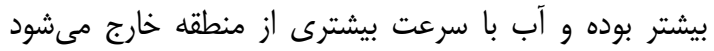

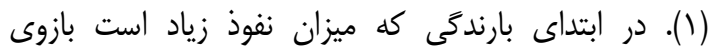

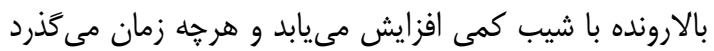

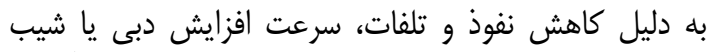

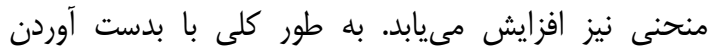

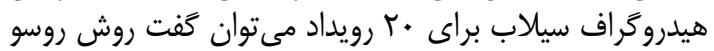

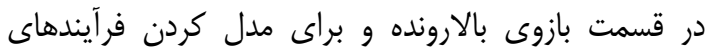

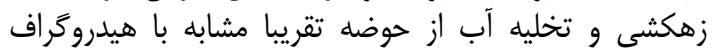

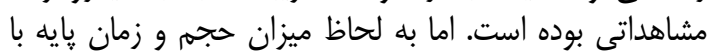

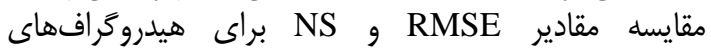

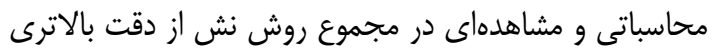

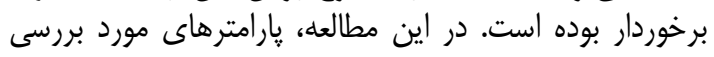

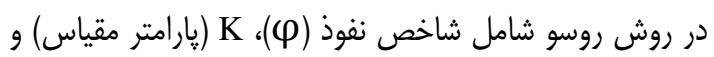

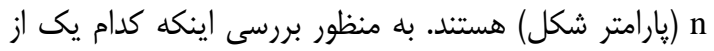

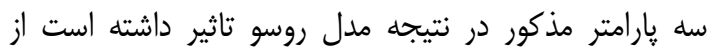

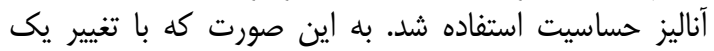

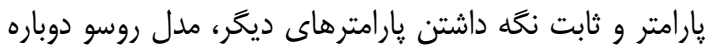

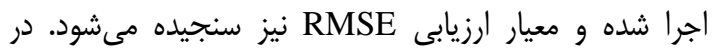

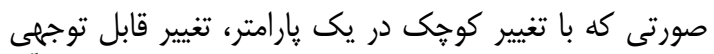

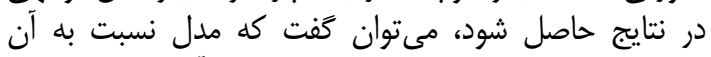
هارامتر حساسيت بيشترى داشته است. طبق آنجه كه دان در شكل

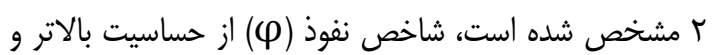

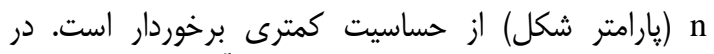

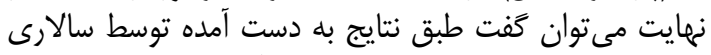

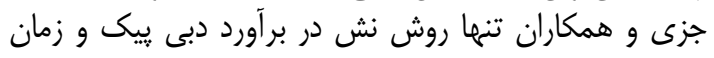


منابع

1. Alizadeh, A. 2010. Principles of applied hydrology. Ferdowsi University of Mashhad. Twenty-Nine (In Persian).

2. Cudennec, C.Y., I. Fouad, J. SumarjoGotot and J. Duchesne. 2004. A geomorphological explanation of the unit hydrograph concept. Hydrological Processes, 18: 603-621(In Persian).

3. Ghahreman, B. and M. Lotfi. 2009. Efficiency of unit hydrograph models in determining the profile of hydrograph of flood. (Applied Research Plan). Northern Khorasan Regional Water Company, 1-117 (In Persian).

4. Ghiasi, P. and M. Roghani. 2004. Efficiency of the instantaneous geomorphological unit hydrograph and its comparison with Schneider.triangular and SCS synthetic hydrographs in the Kashelian basin. Journal of Research and Development, 70: 23-32 (In Persian).

5. Gholami, F., A. Fakherifard and Y. Dinpajooh. 2011. Extracting unit hydrograph geomorphologic based on cascade linear reservoirs (Case study: Lighvan basin). Journal of Irrigation Engineering, 34: 83-93 (In Persian).

6. Heshmatpour, A. 1999.An Investigation of the efficiency of geomorphologic and geomorphologicclimatic instantaneous unit hydrographs in the basin of Kasilian Basin. Master's Degree.Gorgan University of Agricultural Sciences and Natural Resources (In Persian).

7. Hajizadeh, A., M. Mohseni, J. Varvani and M. Vafakhah. 2009. The Relationship of Parameters of Instantaneous Unit Hydrographs with Rainfall and Physiographic Characteristics in Some Watersheds of Iran. Journal of Watershed Research, 83: 22-29 (In Persian).

8. Horton, R.E. 1932. Drainage basin characteristics. Trans Am Geophy Union, 13: 350-361.

9. Iskender, I. and N. Sajikumar. 2016. Evaluation of surface runoff estimation in ungauged watersheds using SWAT and GIUH. International Conference on Emerging Trends in Engineering, Science and Technology, 109-115.

10. Jahanbakhsh, S., B. Rezai, M. Goodarzi, M. Ghafouri, A. Mehdian and M. Mehdian. 2012. Assessment of Clark's time-level method and time-unit hydrograph for estimating flood discharge of Karun basin. Geography and Planning Scientific Journal, 16: 49-66 (In Persian).

11. Narayan, K. 2012. GIS supported geomorphologic instantaneous unit hydrograph (GIUH) of Varuna river basin using Geomorphological Characteristics. International Journal of Advances in Earth Sciences, 2: 68-76.

12. Kerami, F. and M. Ismail Pour. 2014. Estimation of runoff using geomorphologic moment unit hydrograph model. A case study of the Daryan tea basin.Hydrogeomorphology Journal, 1: 157-145 (In Persian).

13. Khaleghi, M. and J. Ghodousi. 2010. Evaluation of the efficiency of geomorphologic unit hydrograph methods in estimating peak flood discharge. Quarterly journal of natural resources science and technology. Fifth year Second Issue, 89-100 (In Persian).

14. Kumar, A. 2014. Geomorphologic Instantaneous Unit Hydrograph Based Hydrologic Response Models for Ungauged Hilly Watershed in India. Water Resources Management, 29: 863-883.

15. Nash, J.E. 1957. The form of the instantaneous unit hydrograph. Institute for Advanced Studies in the Humanities, 45: 114-121.

16. Pirabi, D. and G. Mrinmoy Kr. 2016. Derivation of unit hydrograph from GIS based GIUH. Imperial Journal of Interdisciplinary Research, 2: 555-563.

17. Rahimian, R. and M. Zarea. 1995. Application of geomorphologic unit's hydrograph model for hydrograph synthesis in non-statistical basins. Proceedings of the Third Hydrological Seminar of the Ministry of Energy. Tehran, 3: 203-227 (In Persian).

18. Rodriguez-Iturbe, I., M. Gonzalez-Sanabria and R. Bras. 1982. A geomorphoclimatic theory of the instantaneous unit hydrograph. Water Resources Management, 18: 877-886.

19. Rosso, R. 1984. Nash model relation to Horton order ratios. Water Resources Research, 20: 914- 920.

20. Saeedi, P. and M. NickSokhan. 2015. Estimate the unit geomorphologic unit (GIUH) and unit wavelength hydrograph (WFIUH) in unserved areas. Case study of Ghorve Basin. Journal of Ecohydrology, 2(1): 51-62 (In Persian).

21. Salarijazi, M., A. Adib and M. Akhoundali. 2009. Evaluation of geomorphologic unit hydrograph based on Nash method in basin without rainfall statistics. 5th National Conference on Watershed Management Sciences and Engineering (Sustainable Management of Natural Disasters). Gorgan University of Agricultural Sciences and Natural Resources, 2 to 3 May (In Persian).

22. Salami, W. and I. Ibitoy. 2017. Run off hydrographs using Snyder and SCS synthetic unit hydrograph methods (a case study of selected river in south west Nigeria). Jornal of Ecological Engineering, 18: 25-34.

23. Shirzadi, A., K. Chapi and P. Fathi. 2011. Estimation of synthetic unit hydrograph using regional flood analysis and geomorphologic parameters (case study: Maringan and Kaniyat watersheds of Kordestan). Journal of Agricultural Science and Technology. Water and Soil Science, 15: 231-240 (In Persian). 
24. Shokri, S., A. Behnia and A. Akound ali. 2012. Estimation of Watershed Watershed Using HEC-HMS Homosexual and Geographic Information Model (Case Study: Idenak Watershed). Watershed Management Research. Sari Agricultural and Natural Resources University, 5: 63-80 (In Persian).

25. Snyder, F.F. 1938. Synthetic unit graphs. Trans. Amer. Geophys. Union, 19: 447-454.

26. Strahler, A.N. 1952. Hypsometric (area-altitude) analysis of erosional topography. Geological Society of America Bulletion, 3: 1117-1142

27. Zakizade, F. and A. Talebi. 2015. Investigating the Effects of Methods on the Properties of Parameters and Hydrographs at the Point of View of the Faculty of Agriculture and Natural Resources (Case Study: Monshad Abbas). Watershed Management Research. Sari Agricultural and Natural Resources University, 14: 197-205 (In Persian). 


\title{
The Study of the Effect of Some Used Parameters on Geomorphologic Instantaneous Unit Hydrograph
}

\section{Fatemeh Bayati ${ }^{1}$, Rasoul Mirabbasi ${ }^{2}$, Rouhallah Fatahi $^{2}$ and Mahdi Radfar $^{3}$}

1- Graduated M.Sc. Student, Department of Water Engineering, Shahrekord University Department of Water Engineering, Shahrekord University, (Corresponding author: fatemehbayati88@gmail.com)

2 and 3- Associate Professor and Assistant Professor, Department of Water Engineering, Shahrekord University Department of Water Engineering, Shahrekord University Received March 21, $2018 \quad$ Accepted: March 17, 2019

\begin{abstract}
Estimating the runoff in the basins lacking statistics is always considered by researchers and managers in planning, development and implementation of many projects of water Resource. One of the methods for estimating the runoff is to use geomorphology instantaneous unit hydrograph which estimates the hydrograph of flood based on quantitative geomorphology factors. In this study, first the quantitative geomorphology of the Nash and Rousseau methods including $\mathrm{k}$ (scale parameter), $\mathrm{n}$ (shape parameter) and speed (Meters per second) were obtained. In the next step, instantaneous unit hydrograph was obtained by GIUH method and ultimately flood hydrograph was created for 20 selected events from 1991 to 2007 in kasilian region and compared to the observational hydrographs. Through sensitivity analysis, infiltration Index $(\varphi)$ had higher sensitivity analysis, infiltration Index $(\varphi)$ had higher sensitivity and $n$ (Parameter of shape) were less sensitive. Also, by comparing peak discharge values and computational hydrograph widths in the state where the discharge of hydrographs is 50 and $75 \%$ of peak discharge, observational data revealed that the Rousseau method and lower error than the Nash method and Nash method had a higher accuracy than Rousseau in terms of the volume and time.
\end{abstract}

Keywords: Hydrograoh, Model GIUH, Parameter of shape, Scale parameter 